\title{
Correlation of ultrasound with histopathology for retained products of conception in medically managed abortions
}

\author{
Namita Chopra, Manpreet Kaur*, Manjit Kaur Mohi
}

Department of Obstetrics and Gynecology, G.M.C and Rajindra Hospital, Patiala, Punjab, India

Received: 10 July 2017

Accepted: 14 July 2017

*Correspondence:

Dr. Manpreet Kaur,

E-mail: kalramanpreet@yahoo.com

Copyright: (C) the author(s), publisher and licensee Medip Academy. This is an open-access article distributed under the terms of the Creative Commons Attribution Non-Commercial License, which permits unrestricted non-commercial use, distribution, and reproduction in any medium, provided the original work is properly cited.

\begin{abstract}
Background: Medical abortion uses an anti-progestin, mifepristone (RU486), followed by a prostaglandin (misoprostol). Objective of present study was to correlate findings of transvaginal ultrasound with histopathology for retained products of conception in medical abortions.

Methods: An observational, prospective study was conducted on hundred women with gestation upto 12 weeks who underwent medical abortion with excessive or prolonged post abortal vaginal bleeding. Transvaginal scan followed by uterine evacuation was done under anesthesia, followed by histopathology.

Results: Analysis was done statistically using Pearson Chi- square method. Sixty five percent subjects took MTP pill by unsupervised, self-intake and 35\% on prescription. Among women who took misoprostol in dose of $400 \mathrm{mcg}, 89 \%$ had RPOC on histopathology. At the dose of $800 \mathrm{mcg}, 73.3 \%$ had retained Products of Conception (RPOC) on histopathology. The correlation was found to be statistically non-significant (p value at 5\% was 1.13). Ultrasound showed echogenic mass in the uterine cavity in $62(62 \%)$ women, increased endometrial thickness $\geq 10 \mathrm{~mm}$ in 13 $(13 \%)$, gestational sac with no fetal pole in $6(6 \%)$, blood clots in uterine cavity in $6(6 \%)$, and empty uterine cavity in $3(3 \%)$. Seventy five percent of women had histologically proven RPOC at endometrial cut off of equal to or greater than $10 \mathrm{~mm}$. The sensitivity, specificity, positive and negative predictive value of transvaginal ultrasonography in detection of retained products of conception were $92 \%, 60 \%, 87.3 \%, 71.4 \%$ respectively. The diagnostic accuracy was $84 \%$.

Conclusions: Transvaginal ultrasound for detecting retained products of conception in medically managed abortions has a high sensitivity and positive predictive value and is useful for screening women with clinically suspected incomplete abortion who require further intervention -medical or surgical.
\end{abstract}

Keywords: Medical abortion, RPOC, Transvaginal ultrasound

\section{INTRODUCTION}

The global abortion rate is estimated at 28 per 1000 women of reproductive age but varies within regions. Medical abortion uses an anti-progestin, mifepristone (RU486), followed by a prostaglandin (misoprostol). Medical abortion is preferred from four up to seven or nine weeks. ${ }^{1}$ WHO 2014 has laid down guidelines for dosage of mifepristone and misoprostol- Upto 9 weeks-
Mifepristone $200 \mathrm{mg}$ oral, single dose followed by misoprostol $800 \mathrm{mcg}$ vaginal, buccal or sublingual single dose. From 9 to 12 weeks- mifepristone $200 \mathrm{mg}$ oral single dose followed by Misoprostol $800 \mathrm{mcg}$, then 400 mcg vaginal, followed by sublingual or vaginal every 3 hours up to 5 doses, 36-48 hours after Mifepristone.

Vaginal bleeding with medical abortion generally lasts for 9 days on an average. Woman should seek medical 
advice if there is-prolonged or heavy bleeding (soaking more than two large pads per hour for two consecutive hours) and/or fever lasting for more than 24 hours; A routine follow up visit should be done between 7-14 days. $^{2}$

On ultrasonography, within a week of medical abortion, uterine cavity is rarely empty. Residual clot and decidua may remain and can often include villi. Less than $1 \%$ may show evidence of ongoing pregnancy.

RPOC refers to the persistence of placental and / or fetal tissue in the uterus following delivery, termination of pregnancy or a miscarriage. An endometrial mass is defined as an intrauterine mass distinct from the rest of the endometrium, measurable in 3 dimensions in 2 orthogonal planes. Its echogenicity, size, and location should be looked for. If a separate mass is not visible, the endometrial thickness in the anteroposterior dimension is measured on sagittal midline view. ${ }^{3}$ The endometrial blood clots may give a false positive diagnosis because they appear similar. The histopathology diagnosis of RPOC is made based on the presence of chorionic villi, which indicates persistent placental or trophoblastic tissue.

\section{METHODS}

It was an observational, prospective study conducted on women presenting to the outpatient and emergency department, Obstetrics and Gynaecology, Government Medical College and Rajindra Hospital, Patiala. The study protocol and consent form were reviewed and approved by the ethical committee of the hospital. Written informed consent was taken. One hundred women with period of gestation up to 12 weeks with history of intake of medical abortifacient (mifepristone and/or prostaglandin analogues) with excessive or prolonged post abortal vaginal bleeding were taken up for the study. Transvaginal scan between 10 to 21 days after intake of medical abortifacient was done. Philips HD3 EXPV 225 machine with transvaginal probe of frequency $5 \mathrm{MHz}$ was used.

Presence of any echogenic or heterogeneous material in the endometrial cavity was looked for. In women where endometrium was found to be regular, the endometrial thickness was measured. Uterine evacuation (curettage) was done under short general anesthesia after women's consent. The tissue was sent for histopathological examination and the findings were correlated. Comparisons between groups were made using the Pearson chi-square test for qualitative variables (proportions). The statistical analysis was done using SPSS version 22.00 for the study. Sensitivity, specificity, positive and negative predictive values as well as diagnostic accuracy of ultrasound in detection of retained products of conception was calculated. Relationship between the type, dose and route of drug and incidence of retained products of conception was determined.

\section{RESULTS}

Sixty five percent of women took MTP pill by selfmedication (unsupervised) and $35 \%$ took it on prescription. The mean period of gestation at the time of MTP pill intake was $6.6 \pm 2.2$ weeks. It was observed that $62(62 \%)$ women were moderately anemic, $30(30 \%)$ severely anemic and $8(8 \%)$ were mildly anemic. $86.6 \%$ women with severe anemia, $62.5 \%$ with mild anemia and $53.2 \%$ with moderate anemia, had histologically proven RPOC. $p<0.05$. There is statistically significant association between anemia and RPOC on histopathology. In 59 (59\%) women, tablet misoprostol was taken orally followed by sublingual route in 24 (24\%) and vaginal route in $17(17 \%)$ Most of women who took on prescription took it vaginally $(88 \%)$ or sublingually $(75 \%)$. Chi square statistic is 63.9, p value < 0.00001 , this shows there is statistically significant association between the method and the route of MTP pill intake. The incidence of RPOC on histopathology was $78 \%$ in women who took MTP pill by oral route and $52.9 \%$ in those who took it by vaginal route. Among women who took misoprostol in dose of $400 \mathrm{mcg}, 89 \%$ had RPOC on histopathology, who took misoprostol in dose of $800 \mathrm{mcg}, 73.3 \%$ had RPOC on histopathology. One had taken misoprostol in dose of $1200 \mathrm{mcg}$ and had histologically proven RPOC. On applying z-test, $\mathrm{p}$ value at $5 \%$ is 1.13 . The correlation was not found to be statistically significant.

Transvaginal ultrasound revealed findings of retained products of conception in $81(81 \%)$ women. The findings were- echogenic mass in the uterine cavity in $62(62 \%)$, increased endometrial thickness $\geq 10 \mathrm{~mm}$ in $13(13 \%)$, gestational sac with no foetal pole in $6(6 \%)$, blood clots in uterine cavity in $6(6 \%)$, and empty uterine cavity in $3 \%$.

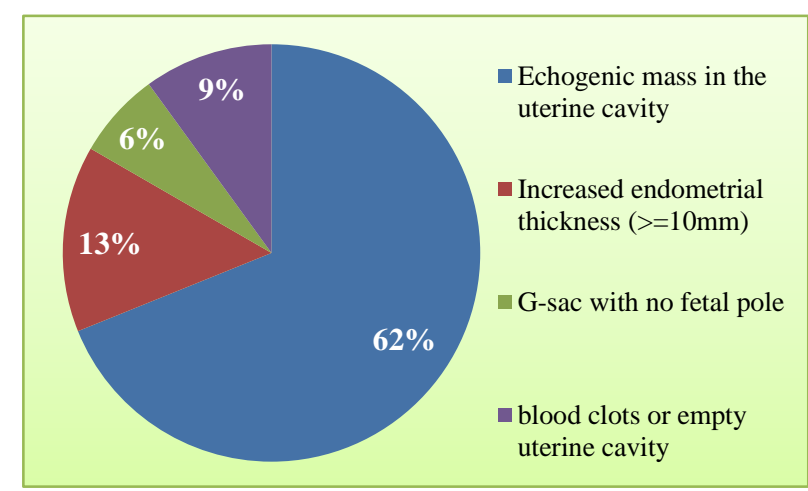

Figure 1: USG findings among subjects $(\mathrm{N}=100)$.

There were 20 women in whom endometrial thickness was regular and could be measured. It was seen that 2 (10\%) had histopathologically proven RPOC at ET less than $6 \mathrm{~mm}, 1(5 \%)$ had RPOC at ET between 6 and $8 \mathrm{~mm}$, $7(35 \%)$ had RPOC at ET between 8 and $10 \mathrm{~mm}, 6(30 \%)$ had RPOC at ET between 10 and $12 \mathrm{~mm}$ and 4 (20\%) had RPOC at ET more than $12 \mathrm{~mm}$. 
Table 1: Correlation between endometrial thickness on USG and histopathologically proven RPOC $(n=20)$.

\begin{tabular}{|lll|}
\hline ET (Endometrial & No. of subjects with & $\%$ \\
\hline thickness) (in mm) & RPOC on histopathology & 10 \\
\hline Less than 6 & 2 & 5 \\
\hline $6-8$ & 1 & 35 \\
\hline $8-10$ & 7 & 30 \\
\hline $10-12$ & 6 & 20 \\
\hline$>12$ & 4 & \\
\hline Total & 20 & \\
\hline
\end{tabular}

It was seen that the ultrasound detected findings were confirmed on histopathology in $89(89 \%)$ and were not correlating with each other in the rest $11(11 \%)$ women. Overall sensitivity of transvaginal ultrasound to detect retained products of conception (RPOC) was $92 \%$ and specificity $60 \%$. It was found to have a positive predictive value (PPV) of $87.3 \%$ and a negative predictive value (NPV) of $71.4 \%$. It was found that USG has a diagnostic accuracy of $84 \%$ in detecting RPOC.

\section{DISCUSSION}

The study showed higher incidence of RPOC in women who took MTP pill by self-medication (77\%) than those who took on prescription (62\%) which is comparable to other studies. In a study by Armo et al, the incidence of RPOC in self intake versus prescribed group was $64.5 \%$ and $35.5 \%$ respectively; and Nivedita et al reported $68.75 \%$ and $31.25 \%$.,5

The incidence of RPOC is $78 \%$ in women who took tablet misoprostol orally which is comparable to the study conducted by Hertzen et al. ${ }^{6}$ This shows that the complete abortion rate is higher in the vaginal misoprostol group as compared to oral group as confirmed by Hertzen et al $78 \%$ in oral group and $8 \%$ in vaginal group and El Rafaey et al $13 \%$ in oral group and $5 \%$ in vaginal group. ${ }^{7}$ The present study shows higher incidence of RPOC with 400 microgram dose of misoprostol $(89 \%)$ than with 800 microgram misoprostol $(73.3 \%)$. This shows that 400 mcg dose might be suboptimal, particularly for period of gestation after 9 weeks. Lille et al also showed relatively higher incidence of RPOC in women taking misoprostol in dose of $400 \mathrm{mcg}$ than in 800 mcg group. ${ }^{8}$

The cut off value of endometrial thickness as suggestive of RPOC in present study is greater than or equal to 10 mm since $75 \%$ of subjects (in whom a regular, thickened endometrium was observed on ultrasound) with this cut off had histologically proven RPOC in the study. Maximum sensitivity was observed at this cut off level. Ustunyurt et al were unable to demonstrate a cut off level, below which RPOC can be accurately excluded. ${ }^{9}$ The negative predictive value was $63 \%$ at cut off level of $10 \mathrm{~mm}$ and $50 \%$ at $8 \mathrm{~mm}$. Sawyer et al found no clear cut off value for endometrial thickness to differentiate between RPOC and decidua in his study. Mc Ewing et al suggested that thickened, heterogeneous endometrium with or without increased Color Doppler flow should be considered part of normal spectrum of post abortion sonographic finding. ${ }^{10}$ Considering these observations, sonographic measurement of endometrial thickness is not a very sensitive and specific parameter for prediction of RPOC.

Using histopathology as confirmatory test, the overall sensitivity of TVS for diagnosis of RPOC in present study is $92 \%$ which is comparable to study conducted by De Vries et al, Matijevic et al and Esmaeillou et al. ${ }^{11-13}$ The overall specificity of TVS is $60 \%$, which is comparable to that in study conducted by Esmaeillou et al, higher than in study conducted by Matijevic et but lower than other studies. ${ }^{12,13}$ This is because of relatively high number of false positive results which might be due to false interpretation of blood clot for RPOC which also looks like echogenic mass on ultrasound or presence of thickened endometrium giving impression of RPOC but proved to be thickened decidua with absence of chorionic villi on histopathology.

The overall positive predictive value of TVS is $87.3 \%$ which is comparable to study conducted by Alcazar et al. The overall negative predictive value is $71.4 \%$ which means that the absence of ultrasound findings excluded RPOC in $71.4 \%$ of cases.

Table 3: Over all sensitivity, specificity, positive and negative predictive value of transvaginal ultrasound in diagnosis of RPOC.

\begin{tabular}{|c|c|c|c|c|}
\hline Study & Sensitivity & Specificity & Positive predictive value & Negative predictive value \\
\hline De Vries et $\mathrm{al}^{11}$ & $85 \%$ & $88 \%$ & $68 \%$ & $92 \%$ \\
\hline Durfee et $\mathrm{al}^{3}$ & $79 \%$ & $89 \%$ & $59 \%$ & $95 \%$ \\
\hline Abbasi et $\mathrm{al}^{14}$ & $78 \%$ & $100 \%$ & $74 \%$ & $82 \%$ \\
\hline Matijevic et al $^{12}$ & $98 \%$ & $33 \%$ & $15 \%$ & $84 \%$ \\
\hline Reeves MF et al ${ }^{15}$ & $40 \%$ & $78.2 \%$ & $29 \%$ & $98.8 \%$ \\
\hline Esmaeillou et al ${ }^{13}$ & $88 \%$ & $68 \%$ & $67 \%$ & $88 \%$ \\
\hline Present Study & $92 \%$ & $60 \%$ & $87.3 \%$ & $71.4 \%$ \\
\hline
\end{tabular}




\section{CONCLUSION}

Transvaginal ultrasound for detecting retained products of conception in medically managed abortions has a high sensitivity and positive predictive value and is a useful tool for screening out patients with clinically suspected incomplete abortion who require further intervention (medical or surgical), thus reducing unnecessary intervention in all such women and avoiding potential surgical morbidity.

Funding: No funding sources Conflict of interest: None declared

Ethical approval: The study was approved by the Institutional Ethics Committee

\section{REFERENCES}

1. Lohr P, Fjerstad M, DeSilva U, Lyus R. British pregnancy advisory service. BMJ. 2014;348:7553.

2. Clinical Practice Handbook for safe Abortion. Abortion, induced-methods, Abortion, inducedstandards, Practice guideline. World Health Organization 2014 available on www.who.int/reproductivehealth

3. Durfee SM, Frates MC, Luong A. The sonographic and color Doppler features of retained products of conception. J Ultrasound Med. 2005;24(9):1181-6.

4. Armo M, Babbar K, Viswas S. Self-medication for Medical Abortion in Rural Scenario: Why to choose Unsafe Way. Int J Sci Stud. 2015;3(6):115-9.

5. Nivedita K, Shanthini F. Is it safe to provide abortion pills over the counter-A study on outcome following self-medication with abortion pills. J Clin Diagn Res. 2015;9(1):OC 01-OC 04.

6. Hertzen HV, Huong NTM, Piaggio G, Bayalag M, Cabezas E, Fang AH et al. Misoprostol dose and route after mifepristone for early medical abortion: a randomized controlled noninferiority trial. BJOG. 2010;117:1186-96.

7. El Rafaey, Rajasekar D, Abdalla M, Calder L, Templeton A. Induction of abortion by Mifepristone and oral or vaginal misoprostol. NEJM. 2016;332(15):983-7.

8. Lille YA, Lefebire P, Roubaix. Ultrasound Monitoring of early medical abortion. Gynecologie Obstetrique Practique. 2007;(4):142-156.

9. Ustunyurt E, Kaymak O, Iskender C, Ustunyurt OB, Celik C, Danisman N. Role of transvaginal sonography in the diagnosis of retained products of conception. Arch Gynecol Obstet. 2008;277:151-4.

10. McEwing RL, Anderson NG, Meates JB, Allen RB, Phillipson GT, Wells JE. Sonographic appearances of the endometrium after termination of pregnancy in asymptomatic versus symptomatic women. J Ultrasound Med. 2009;28(5):579-86.

11. De Vries JI, van der Linden RM, van der Linden HC. Predictive value of sonographic examination to visualize retained placenta directly after birth. J Ultrasound Med. 2000;19(1):7-12.

12. Matijevic R, Knezevic M, Grgic O, Zlodi-Hrsak L. Diagnostic accuracy of sonographic and clinical parameters in the prediction of retained products of conception. J Ultrasound Med. 2009;28(3):295-9.

13. Esmaeillou H, Jamal A, Eslamian L, Marsousi V, Sarvi F, Kokab A. Accurate detection of retained products of conception after first- and secondtrimester abortion by color Doppler sonography. J Med Ultrasound. 2015;23:34-38.

14. Abbasi S, Jamal A, Eslamian L. Role of clinical and ultrasound findings in the diagnosis of retained products of conception. Ultrasound Obstet Gynecol. 2008;32(5):704-7.

15. Reeves MF, Lohar PA, Harwood EJ, Crenin MD. Ultrasound following medical abortion. J Obstet Gynecol. 2008;111:106-12.

Cite this article as: Chopra N, Kaur M, Mohi MK. Correlation of ultrasound with histopathology for retained products of conception in medically managed abortions. Int J Reprod Contracept Obstet Gynecol 2017;6:3372-5. 